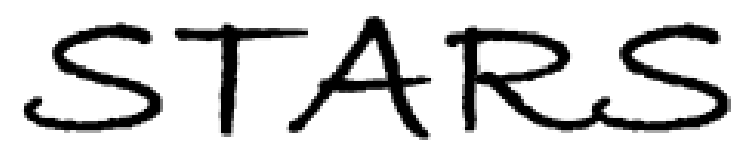

University of Central Florida

STARS

Faculty Bibliography 1990s

Faculty Bibliography

$1-1-1991$

\title{
Shake-Modified Resonant Autoionization In Magnesium
}

S. B. Whitfield

University of Central Florida

J. Tulkki

T. Aberg

Find similar works at: https://stars.library.ucf.edu/facultybib1990

University of Central Florida Libraries http://library.ucf.edu

This Note is brought to you for free and open access by the Faculty Bibliography at STARS. It has been accepted for inclusion in Faculty Bibliography 1990s by an authorized administrator of STARS. For more information, please contact STARS@ucf.edu.

\section{Recommended Citation}

Whitfield, S. B.; Tulkki, J.; and Aberg, T., "Shake-Modified Resonant Autoionization In Magnesium" (1991). Faculty Bibliography 1990s. 371.

https://stars.library.ucf.edu/facultybib1990/371

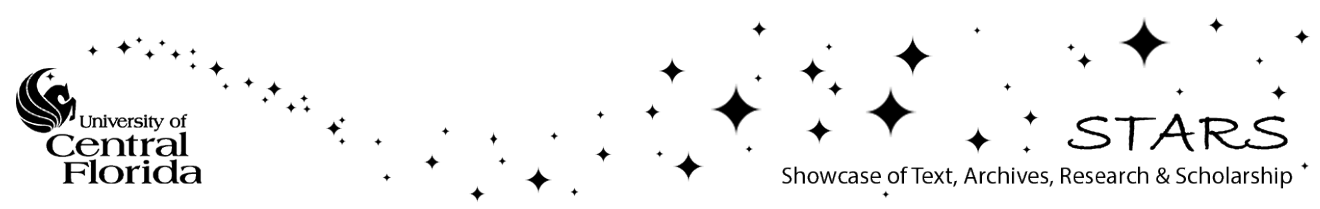




\title{
Shake-modified resonant autoionization in magnesium
}

\author{
S. B. Whitfield \\ Department of Physics, University of Central Florida, Orlando, Florida, 32816 \\ J. Tulkki and T. Åberg \\ Laboratory of Physics, Helsinki University of Technology, 02150 Espoo, Finland
}

(Received 17 April 1991)

\begin{abstract}
Anomalous features in the resonantly excited $2 p$ autoionization spectrum of $\mathrm{Mg}$ are attributed to overlapping shake transitions of the excited bound (spectator) electron. Universal features of the shake spectrum are displayed and related to the post-collision interaction.
\end{abstract}

PACS number(s): $32.80 . \mathrm{Fb}$

The response of atoms to near-threshold photoexcitation has turned out to be a complex phenomenon involving conspicious many-electron effects [1]. As a consequence a large number of satellite peaks usually appear in the spectrum of the photoelectrons [1] and autoionizing electrons $[2,3]$, often with curious energy and angular dependences. In this work we present the first systematic study of a Rydberg electron subject to the radiationless decay of a resonantly excited inner-shell hole state. We have chosen the recently measured $\mathrm{Mg} 2 p$ autoionizing spectrum [4] as an example since it reveals unexpected features which have remained unexplained until now.

The present calculations are based on a resonantscattering-theoretical formulation of the decay of metastable states [5]. The basic expression of the differential cross section is exactly the same as the one which has successfully been used for the description of the angle-averaged postcollision-interaction (PCI) effect [6-8]. We use a relativistic formulation of PCI since all calculations have been made using the multichannel multiconfiguration Dirac-Fock (MMCDF) method [9]. This does not exclude states with strong $L S$ coupling, since they are superpositions of $j j$-coupled states. The mixing coefficients are obtained in such a case using the configuration-interaction (CI) technique.

Basically the autoionization following photoexcitation should be treated as a one-step process mediated by a complete set of intermediate discrete and continuous onehole states $[5,6]$. The final-state scattering wave function pertains to one electron in the continuum and another electron in either a discrete excited state or a lowenergy continuum state. The ionic core is thus doubly charged in the final states which interact with the intermediate, states through the full Hamiltonian. It can be seen from the general transition-matrix element that the major contribution to the cross section must come from a final-state phase-space part in which one of the electrons nearly moves with the characteristic autoionizing energy $[5,7]$. If this energy is large in comparison with the excitation energy of the other electron, then it follows from expressions which involve nonorthogonal orbitals in the appropriate Hamiltonian matrix element [10] that there is a factorization of the many-electron interaction am- plitude into an overlap element $\left\langle n l j \mid n^{\prime} l j\right\rangle$ and the autoionization probability amplitude. In this overlap element $\left|n^{\prime} l j\right\rangle$ is the orbital of an excited electron in the presence of the intermediate hole $n_{i} l_{i} j_{i}$, and $\mid n l j>$ an orbital which describes an excited electron in the field of the doubly charged final core. Either or both orbitals could be in the continuum. This is the approximation we shall use below in the autoionization cross section, Eq. (2). Factorization most likely breaks down for $n^{\prime}$ close or equal to the principal quantum number of the outermost occupied shell or for low characteristic energies.

In the following an excited bound electron described by the $\mid n^{\prime} l j>$ wave function will be referred to as a spectator electron. If $n>n^{\prime}$ we have shakeup or shakeoff, if $n<n^{\prime}$ we have shakedown, and for $n=n^{\prime}$ we have a pure spectator transition. The shake probability is given by the square of the overlap matrix element $\left\langle n l j \mid n^{\prime} l j\right\rangle$.

According to energy conservation we have

$$
\omega+E_{0}=E^{(+)}+E_{\mathrm{exc}}=E^{(2+)}+\varepsilon_{A}+\varepsilon,
$$

where $\omega$ is the photon energy and $E_{0}$ the total groundstate energy of the atom. In the final state the autoionizing electron has the energy $\varepsilon_{A}$ and the excited electron the energy $\varepsilon=-\varepsilon_{n l j}$ with respect to the total energy $E^{(2+)}$ of the doubly charged core. According to Eq. (1) the excess energy $E_{\text {exc }}$ is negative, whenever $\omega$ is tuned below $E^{(+)}-E_{0}$, where $E^{(+)}$is the total energy of an ion with a hole in the subshell $n_{i} l_{i} j_{i}$. Without a spectator electron the kinetic energy of the autoionizing electron would be $\varepsilon_{A}^{0}=E^{(+)}-E^{(2+)}$.

Under the assumptions made above the theory of shake-modified resonant autoionization is identical to that of the lowest-order theory of PCI including the recapture of the photoelectron [8]. The relativistic cross section for autoionization in atomic units is

$$
\begin{aligned}
\frac{d \sigma}{d \varepsilon}=\frac{\pi}{3 \omega \alpha} \sum_{l j, l_{A} j_{A}}[ & \Gamma_{l_{A} j_{A}}(\varepsilon)\left|<\varepsilon^{\prime} l j\right| \tau_{0}>\left.\right|^{2} \\
& \left.+\Gamma_{l_{A} j_{A}}\left(\varepsilon^{\prime}\right)|<\varepsilon l j| \tau_{0}>\left.\right|^{2}\right],
\end{aligned}
$$

where $\varepsilon^{\prime}=E_{\text {exc }}+\varepsilon_{A}^{0}-\varepsilon$. In Eq. (2) $\Gamma_{l_{A} j_{A}}$ are the partial autoionizing rates and 


$$
\begin{aligned}
& \mid \tau_{0}> \\
& \quad=\int \frac{\left|\tau \mathrm{lj}><\left[\mathrm{n}_{\mathrm{i}} \mathrm{l}_{\mathrm{i}} \mathrm{j}_{\mathrm{i}}\right] \tau \mathrm{lj}, \mathrm{J}=1\right|\left|\sum_{\nu} \alpha \cdot \mathbf{A}\left(\mathbf{r}_{\nu}\right)\right| \mid \mathrm{J}=0>}{\mathrm{E}_{\mathrm{exc}}-\tau+\mathrm{i}(\Gamma / 2)} \mathrm{d} \tau
\end{aligned}
$$

involves the reduced $E 1$ dipole many-electron matrix element in the Coulomb (velocity) gauge, and the width $\Gamma$ of the hole state $\left[n_{i} l_{i} j_{i}\right][8,11]$. If $\omega$ is tuned to one of the resonances below $E^{(+)}-E_{0}$, then $E_{\text {exc }} \simeq-\bar{\varepsilon}_{n^{\prime} l j}$ which is given with respect to the total energy $E^{+}$of the ion with the hole $n_{i} l_{i} j_{i}$. Consequently the main contribution to the cross section comes from the second term in the square brackets, and as long as $\Gamma \ll \bar{\varepsilon}_{n^{\prime} l j}-\bar{\varepsilon}_{n^{\prime}+1 l j}$ only one state contributes to $\left|\tau_{0}\right\rangle$, namely, that $\left|n^{\prime} l j\right\rangle$ for which $\tau \simeq E_{\text {exc }}$. This is the approximation we shall use in the following.

Our test case consists of $2 p_{j}\left(j=\frac{1}{2}, \frac{3}{2}\right) \rightarrow n^{\prime} l j(l=0,2)$ $E 1$ transitions followed by $2 p^{5} 3 s^{2} n^{\prime} l \rightarrow 2 p^{6} n l \varepsilon_{A} l_{A}$ autoionization in $\mathrm{Mg}$ [4]. The initial, intermediate, and final core-state wave functions were optimized separately without including the orbitals of the excited electron. These orbitals were created by keeping the core frozen, however, the exchange interaction was included fully. The Lagrange-multiplier technique was employed to ensure orthogonality in the intermediate and final states, respectively.

The calculations were not only carried out by using a single $j j$-coupled configuration for each intermediate and final state but also in the intermediate-coupling scheme by mixing $j j$-coupled states coming from the same nonrelativistic parent configuration and having the same total angular momentum. This had, however, a minor effect on the orbitals which were used to generate the total shake probabilities. Since we always sum over the finestructure components of excited electrons, the use of pure $j j$ coupling was found to be entirely adequate in spite of the fact that the spectator many-electron states are almost pure $L S$ states.

For $\mathrm{Mg}[2 p], \Gamma=1.00 \pm 0.25 \mathrm{meV}$ [12]. This means that it is legitimate to use the one-state approximation for $\mid \tau_{0}>$ in Eq. (3) up to states with $n^{\prime}$ about 30, for which the energy spacings of adjacent levels are close to $1 \mathrm{meV}$. Figure 1 shows the behavior of the shake probability $P_{n n^{\prime}}=|<n l| n^{\prime} l>\left.\right|^{2}(l=0,2)$ as a function of $n$ and $n^{\prime}$ for $n^{\prime} \leq 13$. Shake probabilities for discrete transitions are plotted in the form of histogram blocks of height $P_{n n^{\prime}}$. The shakeoff was also calculated for a number of intermediate $n^{\prime} s$ and $n^{\prime} d$ states, but was found to be small. The figure displays the following universal features of the shake probability of the spectator electron.

(i) The probability $P_{n^{\prime} n^{\prime}}$ that the spectator electron keeps its quantum number $n^{\prime}$ during autoionization oscillates as a function of $n^{\prime}$. For a given $n^{\prime}, P_{n n^{\prime}}$ also oscillates as a function of $n$. This is an interference effect, related to the change in the number of nodes in the wave functions.

(ii) Shakeup dominates over shakeoff and shakedown. As $n^{\prime}$ increases the maximum of the shakeup distribution shifts from $n=n^{\prime}+1$ toward higher $n$ values. As a consequence of the centrifugal barrier this effect is more pronounced for $l=2$ than for $l=0$. The strongest shakedown transition is not necessarily from $n^{\prime}$ to $n^{\prime}-1$. The strong dependence of the autoionizing rates on the principal quantum number of the spectator electron has been previously noticed in the case of the decay of the $\operatorname{Ar} 2 p^{5} n^{\prime} d$ states [3].

As the energy spacing of adjacent levels becomes less than $\Gamma$, that is $E_{\text {exc }} \gtrsim-\left(\frac{1}{2}\right) \Gamma^{2 / 3}$, more and more states contribute to the formation of $\left|\tau_{0}\right\rangle$ in Eq. (3). For $E_{\text {exc }} \gtrsim 2 \Gamma, \mid \tau_{0}>$ is predominantly a superposition of continuum states which results in a shake spectrum dominated by shakedown [6-8]. Outside the ionic core the real and imaginary parts of $\mid \tau_{0}>$ attain the form of a damped oscillating wave as a function of the radial distance $r$ from the nucleus [6]. The damping factor $\exp \left(-r / r_{0}\right)$, where $r_{0} \simeq(2 / \Gamma)\left(2 E_{\text {exc }}\right)^{1 / 2}$, stems from the asymptotic dependence of the continuum wave functions $\mid \tau l j>$ on $r$ in Eq. (3). As $\varepsilon$ increases from negative to positive values the overlap integral of the increasingly oscillating final-state wave function $\mid \varepsilon l j>$ with $\mid \tau_{0}>$ is not only affected by the asymptotic phase matching but also by the damping factor in combination with the change of the ionic charge. As a consequence the probability $|<\varepsilon l j| \tau_{0}>\left.\right|^{2}$ tends to reach the maximum before $\varepsilon$ exceeds $E_{\text {exc }}$ and to concentrate in the energy region below $E_{\text {exc }}$. Recapture [8] and shakedown $[6,7]$ of the spectator electron, which has now become the photoelectron, are thus favored at the expense of shakeoff to continuum states with $\varepsilon>E_{\text {exc }}$. The maximum of the photoelectron line is shifted towards kinetic energies below $E_{\text {exc }}$ and the Lorentzian line shape is distorted. The quantum-mechanical picture is entirely consistent $[6,7]$ with the semiclassical description of PCI [13], where the photoelectron slows down due to the in-
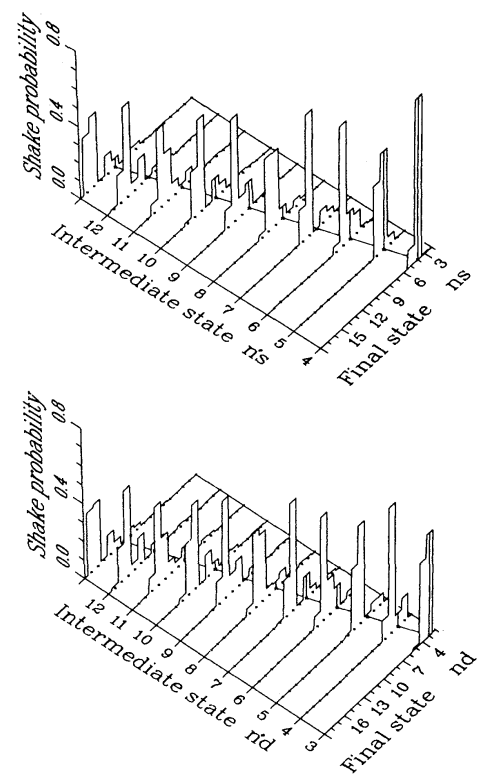

FIG. 1. Shake probability as a function of the principal quantum numbers of the intermediate and final $l=0,2 \mathrm{spec}-$ tator states. The diagonal line traces the probability that the spectator electron keeps its principal quantum number during the decay. 
crease of the attractive charge of the core by one unit during the autoionization process. This shift is compensated by an increase in the velocity of the autoionizing electron.

As indicated above the transition regime in which shake-modified spectator emission with more shakeup than shakedown goes over into PCI distorted emission with more shakedown than shakeoff approximately covers $E_{\text {exc }}$ ranging from $-\left(\frac{1}{2}\right) \Gamma^{2 / 3}$ to $2 \Gamma$. If $\Gamma \simeq 1 \mathrm{meV}$, as in the $\mathrm{Mg}[2 p]$ case, this amounts to $-15 \mathrm{meV} \lesssim E_{\text {exc }} \lesssim 2$ $\mathrm{meV}$ which is too narrow to be experimentally accessible. It would thus be of interest to resonantly excite this threshold region with higher resolution than previously in a case [14] for which $\Gamma$ is large enough to produce an experimentally accessible transition regime.

In a recent measurement of electron spectra which result from resonant excitation of $[2 p] 3 s^{2} n^{\prime} s, d\left(n^{\prime} \geq 4\right)$ autoionizing states of $\mathrm{Mg}$ several satellite lines were observed [4]. On the binding energy scale they appear on the high-energy side of the $3 s$ photoelectron line indicating that the $\mathrm{Mg}^{+}$ion is left in states that lie higher in energy than the $[3 s]^{2} S$ ground state. For excitation to a given $n^{\prime} s$ or $n^{\prime} d$ Rydberg state, the $[2 p] n^{\prime} s, d \rightarrow$ $\left[3 s^{2}\right] n^{\prime} s, d$ spectator lines were observed in each case, but these lines were not necessarily the strongest. The appearance of the other satellite lines could be explained by arguments based on shakeup or shakedown of the $n^{\prime} s, d$ spectator electrons, and on correlation involving $n^{\prime}=3$ electrons. A notable exception was the $\hbar \omega=57.34(3) \mathrm{eV}$ resonance excitation. The tuning was assumed to result in transitions from the ground state to the $\left[2 p_{1 / 2}\right] 3 s^{2} 6 d$ state, which shakes most strongly into the $\left[3 s^{2}\right] 8 d$ state of the ion. Yet the strongest line seemed to correspond to the $\left[3 s^{2}\right] 11 d$ final state, leading to the emission of an electron with the same kinetic energy as the $\left[2 p_{1 / 2}\right] \rightarrow\left[3 s^{2}\right]$ Auger transition [4]. In the following we shall see that a complete interpretation of this spectrum requires consideration of the $2 p$ spin-orbit splitting as well as shakeup and shakedown of electrons in several spectator states.

Table I shows that there are four different $2 p$-hole Rydberg states lying within $70 \mathrm{meV}$ of each other, all in the vicinity of the 57.34-eV resonant photon energy. Since the bandwidth of the synchrotron radiation in the experiment was approximately $80 \mathrm{meV}$, all of these states could be reached. Thus, any reliable interpretation of the experimental spectrum must take into account all four res- onant excitations and their subsequent decay. Two sets of excitation energies are shown; the theoretical values were used in our analysis. The corresponding oscillator strengths are shown in the last column. It is seen that the strengths are comparable in magnitude.

In order to make a meaningful comparison with the experimental spectrum the cross section Eq. (2) must be convoluted with the spectral function of the monochromator and instrumental function of the electron analyzer. These were assumed to be Gaussian functions with a full width at half maximum equal to 80 and $200 \mathrm{meV}$, respectively [4]. As explained previously we used the onestate approximation in $\left|\tau_{0}\right\rangle$, Eq. (3), since $\Gamma \simeq 1 \mathrm{meV}$ for the $2 p$ hole. The shakeup and shakedown transitions which had any significant strength were included for each of the four intermediate states listed in Table I. The result of our calculation over the photon energy range from 57.1 to $57.6 \mathrm{eV}$ is shown in Fig. 2. It should be stressed that each electron energy distribution is a combination of excitation and decay probabilities including shakeup and shakedown, in accordance with Eqs. (2) and (3). The electron spectrum thus corresponds to several final $\left[3 s^{2}\right] n s, d$ states some of which do not contribute very much depending on the photon energy.

Figure 2 demonstrates that the variation of the photon energy would change drastically the shape of the electron emission spectrum. Our result clearly shows that the excitation part including the width of the resonance cannot be neglected in a detailed analysis of resonantly excited autoionizing states, in accordance with Eq. (3).

Figure 3 shows a comparison between theory and experiment. The agreement is excellent provided the photon energy is chosen to be equal to $57.31(2) \mathrm{eV}$ which is within the error limits of the experimental energy. It should be noted that the only experimental parameters used in the construction of cross section, Eq. (2), are the $2 p_{1 / 2,3 / 2}$ ionization energies [15], and the width of the corresponding hole states [12]. The $\left[2 p_{1 / 2}\right] \rightarrow\left[3 s^{2}\right]$ Auger transition does not play any role in our interpretation since it only appears as a consequence of the presence of the second harmonic in the dispersed synchrotron radiation.

In conclusion, we have presented the first systematic study of shake transitions of a spectator electron subject to the decay of resonantly excited autoionizing states. The underlying resonant-scattering theory reveals the re-

TABLE I. Energies and oscillator strengths of $2 p$-hole Rydberg states used in the construction of Figs. 2 and 3.

\begin{tabular}{|c|c|c|c|c|}
\hline \multirow[b]{2}{*}{ Configuration } & \multicolumn{4}{|c|}{ Excitation energy $(\mathrm{eV})$} \\
\hline & Expt. $^{a}$ & Theory ${ }^{\mathrm{b}}$ & Oscillator strength ${ }^{c}$ & \\
\hline & {$\left[2 p_{3 / 2}\right] 9 s_{1 / 2}$} & $57.281(5)$ & 57.292 & 0.0137 \\
\hline & {$\left[2 p_{1 / 2}\right] 7 s_{1 / 2}$} & $57.300(5)$ & 57.335 & 0.0181 \\
\hline & {$\left[2 p_{3 / 2}\right] 8 d_{3 / 2,5 / 2}$} & $57.324(5)$ & 57.326 & 0.0261 \\
\hline & {$\left[2 p_{1 / 2}\right] 6 d_{3 / 2,5 / 2}$} & $57.347(5)$ & 57.422 & 0.0287 \\
\hline
\end{tabular}

\footnotetext{
a Reference [15]. The $\left[2 p_{3 / 2}\right] 9 s, 8 d$ energies which do not appear in the photoabsorption data (Ref. [15]) were determined by subtracting the $2 p$ spin-orbit energy from the corresponding experimental $2 p_{1 / 2}$ energies.

b The calculated eigenenergies of the spectator electron were substracted from the experimental ionization energies (Ref. [15]).

${ }^{c}$ Calculated using the expression for the reduced matrix element in Eq. (3).
} 


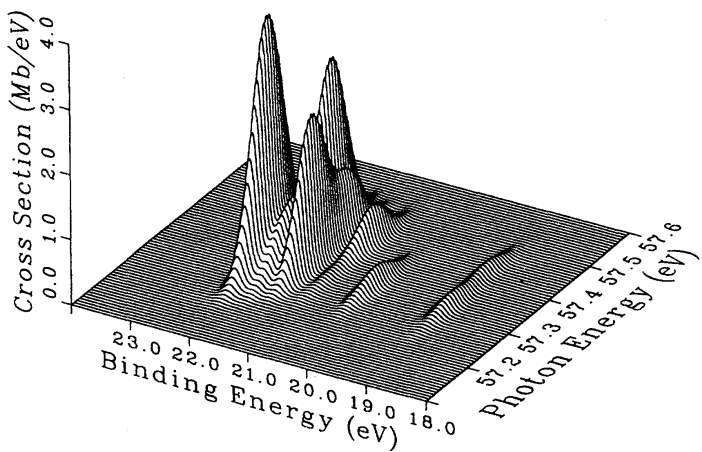

FIG. 2. Cross section (2), after convolution with the spectral function of the monochromator and instrumental function of the electron analyzer (see text), as a function of the binding energy in the 57.1 to $57.6-\mathrm{eV}$ photon energy range.

lationship between the shake transitions and the post collision interaction. As an application, we have completed the analysis of electron emission spectra following the decay of $\left[2 p_{1 / 2,3 / 2}\right] 3 s^{2} n^{\prime} s, d$ states of $\mathrm{Mg}$ in the vicinity of the $57.34-\mathrm{eV}$ resonant photon excitation. All anomalous features have been explained. Our findings should be useful for future planning of synchrotron-radiation experiments on the dynamics of resonantly excited subthreshold inner-shell hole states.

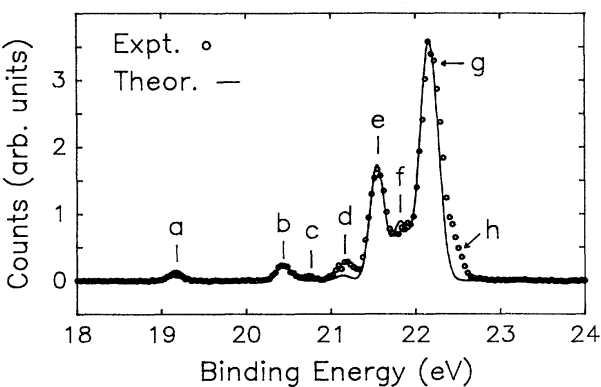

FIG. 3. A comparison between experimental (open circles, Ref. 4) and theoretical (solid line) electron emission spectra. The spectra are normalized at the maximum of peak $g$, and the theoretical spectrum corresponds to a photon energy of $57.31 \mathrm{eV}$ in Fig. 2. The intermediate spectator states are given in Table I and the final spectator states are identified as follows: $a=4 d+5 s, b=5 d+6 s, c=5 p$ (not included in our calculation), $d=6 d+7 s, e=7 d+8 s, f=8 d+9 s$, $g=10 d+11 s+11 d+12 s$. The $9 d+10 s$ line is too weak to appear as an isolated feature. The experimentally observed broadening of the high-energy flank of peak $g$ (marked $h$ ) is due to the $\left[2 p_{3 / 2}\right] \rightarrow\left[3 s^{2}\right]$ Auger line, produced by the second harmonic of the dispersed synchrotron radiation.

We are grateful to C. D. Caldwell and M. O. Krause for a critical reading of the manuscript and constructive comments. This work was supported by the National Science Foundation under Grant No. PHY-8907286 and by the Academy of Finland.
[1] Several review articles have recently appeared in conference proceedings: $M$. O. Krause, in Proceedings of the 8th International Conference on VUV Physics, edited by P.-O. Nilsson and J. Nordgren [Phys. Scr. T17, 146 (1987)]; D. A. Shirley, U. Becker, P. A. Heimann, and B. Langer, J. Phys. (Paris) C9, 427 (1987); U. Becker, in The Physics of Electronic and Atomic Collisions, XVI International Conference on the Physics of Electronic and Atomic Collisions, edited by A. Dalgarno, R. S. Freund, P. M. Koch, M. S. Lubell, and T. B. Lucarto, AIP Conf. Proc. No. 205 (AIP, New York, 1990), p. 162; in X-Ray and Inner-Shell Processes, Fifteenth International Conference on X-Ray and Inner-Shell Processes, edited by T. A. Carlson, M. O. Krause, and S. T. Manson, AIP Conf. Proc. No. 215 (AIP, New York, 1990), p. 621; U. Becker and D. A. Shirley, in Proceedings of the 9th International Conference on VUV Physics, edited by D. A. Shirley and G. Margaritondo [Phys. Scr. T31, 56, (1990)].

[2] B. Kämmerling, B. Krässing, and V. Schmidt, J. Phys. B 23, 4487 (1990), and references therein.

[3] M. Meyer, E. v. Raven, B. Sonntag, and J. E. Hansen, Phys. Rev. A 43, 177 (1991).

[4] S. B. Whitfield, C. D. Caldwell, and M. O. Krause, Phys. Rev. A 43, 2338 (1991).
[5] T. Åberg, Phys. Scr. 21, 495 (1980).

[6] J. Tulkki, G. B. Armen, T. Åberg, B. Crasemann, and M. H. Chen, Z. Phys. D 5, 241 (1987).

[7] G. B. Armen, J. Tulkki, T. Åberg, and B. Crasemann, Phys. Rev. A 36, 5606 (1987).

[8] J. Tulkki, T. Åberg, S. B. Whitfield, and B. Crasemann, Phys. Rev. A 41, 181 (1990).

[9] J. Tulkki, Phys. Rev. Lett. 62, 2817 (1989).

[10] T. Åberg and G. Howat, in Encyclopedia of Physics, edited by S. Flügge and W. Mehlhorn (Springer, Berlin, 1982), Vol. 31, p. 469.

[11] In the following square brackets will be used to denote hole states.

[12] M.O Krause and J. H. Oliver, J. Phys. Chem. Ref. Data 8329 (1979).

[13] A. Niehaus, J. Phys. B 10, 1845 (1977); A. Niehaus and C. J. Zwakhals, ibid. 16, L135 (1983); A. Russek and W. Mehlhorn, ibid. 19, 911 (1986); P van der Straten, R. Morgenstern, and A. Niehaus, Z. Phys. D 8, 35 (1988).

[14] G. B. Armen, T. Åberg, J. C. Levin, B. Crasemann, M. H. Chen, G. E. Ice, and G. S. Brown, Phys. Rev. Lett. 54, 1142 (1985).

[15] D. L. Ederer, T. B. Lucatorto, and G. Mehlman, J. Opt. Soc. Am. 69, 520 (1979) and references therein. 